\title{
Intraperitoneal antineoplastic drug delivery: experience with a cyclophosphamide, vincristine and prednisolone protocol in cats with malignant lymphoma
}

Keywords

cat, chemotherapy, intraperitoneal, lymphoma, safety
Correspondence address: E. Teske

Department of Clinical Sciences of Companion Animals, Veterinary Faculty Utrecht University PO Box 80.154, 3508 TD Utrecht, The Netherlands e-mail: e.teske@uu.nl

\author{
E. Teske ${ }^{1}$, A. J. van Lankveld ${ }^{1}$ and G. R. Rutteman ${ }^{1}$ \\ Department of Clinical Sciences of Companion Animals, Veterinary Faculty, Utrecht University, Utrecht, \\ The Netherlands
}

\begin{abstract}
In this retrospective study, the efficacy and safety were examined for an intraperitoneal chemotherapy protocol-cyclophosphamide, vincristine and prednisolone (IP-COP) in 26 cats with malignant lymphoma. Certainly in cats fiercely resisting IV administration the IP route is a more practical method, safer for the administrator and less stressful for the cat. Complete remission (CR) rate was $76.9 \%(n=20)$. Median duration of first remission was 421 days. Estimated 1- and 2-year disease free period were 67.1 and $48.0 \%$, respectively. Median duration of survival was 388 days and estimated overall 1- and 2-year survival periods were 54.7 and $46.9 \%$ respectively. Young cats had a more favourable prognosis. Reaching CR was essential for long-term survival. No specific IP-related adverse events (AE) were seen. AE were generally scored as mild and were not excessively abundant. These results indicate that the IP route is a safe and effective alternative for the administration of COP protocol chemotherapeutics.
\end{abstract}

\section{Introduction}

Malignant lymphoma (ML) is one of the most common malignancies seen in the cat in the Utrecht University Clinic for Companion Animals (UUCCA). ${ }^{1}$ Reported estimate incidence rates vary from 160 to 200 per 100000 individuals. ${ }^{1,2}$ Incidence of $\mathrm{ML}$ and prevalence of the different anatomical forms greatly vary in different populations, separated geographically or in time. ${ }^{3,4}$ In The Netherlands, for instance, the most frequent anatomic form is the mediastinal one., ${ }^{1,5}$ Causes for these differences may be variation in feline leukaemia virus (FeLV) status and breed differences in the cat population.

In the 1960s, these tumours were often associated with retroviruses, predominantly FeLV infection.
The incidence of FeLV in The Netherlands is one of the lowest in the world $(<0.3 \%),{ }^{1,4}$ due to decennia long policy of testing and eliminating in the breed cat population. ${ }^{1,2}$ The percentage of FeLV positive ML in The Netherlands is also very low $(<8 \%){ }^{1}$ Nevertheless, the incidence of feline ML remains high.

The treatment of choice when assessing ML is chemotherapy. Many variations in treatment protocols exist. Protocols vary in efficiency depending on the type of lymphoma and its susceptibility to the agents. Combination protocols tend to be more effective than single-agent ones and long maintenance is required. ${ }^{3,7}$ At the UUCCA, a multidrug protocol is used consisting of cyclophosphamide, vincristine and prednisolone, the COP protocol, already proven to be effective. ${ }^{1,8}$ 
Remission rates previously were found to differ per anatomic location with an overall remission rate of approximately $75 \%$. $^{1,8}$

Although the IV-based COP protocol is effective, this route of administration in our experience often was very hard to execute in frightened and aggressive cats. This was mainly because the cats resist to being restrained and injected intravenously. Although sedation may then be considered, this adds to the burden of treatment and costs, leading us to investigate intraperitoneal (IP) administration. Arguments to consider this route as potentially effective were found in literature demonstrating high efficacy for other IP protocols in experimental animals and the human. ${ }^{9,10}$ The goal was improving safety for the administrator and reducing stress for the animal. Because of its apparent success many more cats have been treated with this IP-COP protocol. The aim of this study was to investigate the efficacy and toxicity of this new route of administration.

\section{Material and methods}

\section{Animals}

All cats included in this study $(n=26)$ were referred to the Utrecht University Clinic for Companion Animals (UUCCA) and were all treated according to the COP protocol with solely IP administration of the chemotherapeutics. Cats that were partially treated with IP and IV injections were excluded.

Diagnosis of ML was always confirmed prior to treatment by either cytology or histology of a biopsy of an organ or an excised lymph node. Other serious medical illnesses were absent in these animals.

\section{Data collection}

This study was conducted by assessing the clinic database (Vetware ${ }^{\circledR}$, in use since 2004) of the UUCCA from 2004 to 2011. Information that was obtained from this database included age, breed, gender, the exact diagnose, medical history, drug administration reports and reports of clinical state and adverse events (AE; vomiting, diarrhoea, anorexia, obstipation, fever, pain, hair loss or dullness). In addition, possible pretreatment with corticosteroids within 3 weeks prior to chemotherapy was noted. As not all tumours in these cats were staged, stage was not included as part of the prognostic criteria study. FeLV/FIV status was evaluated in 15 cats with an ELISA test.

Cats were classified according to six anatomical categories: nasal lymphoma, mediastinal lymphoma, alimentary lymphoma, lymphoma of the liver, peripheral lymphoma and miscellaneous types of lymphoma.

Long term follow-up (i.e. after completion of treatment) for cases where disease free period (DFP) and survival time was no longer listed in the database was conducted by phoning the owners.

\section{Treatment}

All cats that were eligible for this study were treated according to a modified COP protocol (Table 1). The original protocol was described by Cotter et al. ${ }^{8}$ The modification lies in the fact that all injections are given IP. Induction of remission was performed with vincristine $\left(0.75 \mathrm{mg} / \mathrm{m}^{2} ; 0.1 \mathrm{mg} / \mathrm{mL}\right.$; IP $)$ weekly in weeks 1 , 2, 3 and 4 , and cyclophosphamide $\left(300 \mathrm{mg} / \mathrm{m}^{2} ; 20 \mathrm{mg} / \mathrm{mL}\right.$; IP) weekly in weeks 1 and 4 , in separate administrations, combined with a daily oral dose of prednisolone $\left(50 \mathrm{mg} / \mathrm{m}^{2}\right)$. IP

Table 1. Chemotherapy protocol used in this study

\begin{tabular}{|c|c|c|c|c|c|c|c|c|c|c|c|c|c|c|c|c|}
\hline \multirow[b]{2}{*}{ Agent } & \multirow[b]{2}{*}{ Dose } & \multirow[b]{2}{*}{ Route } & \multicolumn{14}{|c|}{ Treatment week } \\
\hline & & & 1 & 2 & 3 & 4 & 5 & 6 & 7 & 8 & 9 & 10 & 13 & 16 & $\ldots^{a}$ & 52 \\
\hline Vincristine & $0.75 \mathrm{mg} / \mathrm{m}^{2}$ once & $\mathbb{I P}$ & $x$ & $x$ & $x$ & $x$ & & & $x$ & & & $x$ & & $x$ & & $x$ \\
\hline Cyclophosphamide & $300 \mathrm{mg} / \mathrm{m}^{2}$ once & IP & $x$ & & & $x$ & & & $x$ & & & $x$ & & $x$ & & $x$ \\
\hline Prednisolone & $50 \mathrm{mg} / \mathrm{m}^{2}$ daily & $\mathrm{PO}$ & $x$ & $x$ & $x$ & $x$ & $x$ & $x$ & $x$ & $x$ & $x$ & $x$ & $x$ & $x$ & $x$ & $x$ \\
\hline L-Asparginase $\mathrm{b}^{\mathrm{b}}$ & $400 \mathrm{IU} / \mathrm{kg}$ once & SC & $x$ & & & & & & & & & & & & & \\
\hline
\end{tabular}

aPrednisolone is continued daily throughout the complete period, cyclophosphamide and vincristine once every three weeks.

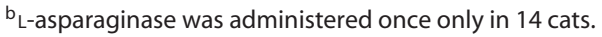


injection was performed in a vertically fixated cat $1 \mathrm{~cm}$ lateral of the navel during a 30- to 40-second period, using a butterfly needle which was gently injected into the abdominal cavity. When no fluid, either blood or urine, was noticed a Tevadaptor ${ }^{\circledR}$ (TEVA Medical Ltd, Netanya, Israel) system was connected for administration of hazardous drugs. The butterfly needle was flushed with saline after the chemotherapy administration before being retracted. In 14 cats, a single administration of L-asparaginase (400 IU/kg, SC) was given a few days prior to this induction phase. After the first 4 weeks of induction therapy, remission was maintained with vincristine and cyclophosphamide injections (IP) given on the same day with 3-week interval for the duration of 1 year or until relapse of the illness. Dosing is the same as in the induction phase. Prednisolone is continued daily until relapse or for 1 year in remission. After this 1 year remission, the dose is gradually decreased and stopped in a 3-week period.

\section{Evaluation of response}

Response was only evaluated once all eligibility criteria were satisfied. At each treatment the cats were evaluated by the oncologists. Reports of the findings were made in Vetware ${ }^{\circledR}$. Response was evaluated by clinical appearance and measurement of tumour volume. In cats with an alimentary or mediastinal tumour, abdominal ultrasounds and thoracic radiographs were used, respectively, to determine tumour volume and to evaluate response. In nasal lymphomas, evaluation was aided by rhinoscopy. Other types of lymphomas were evaluated through physical examination prior to treatment.

Cats were considered to have no response or progression when there occurred a less than 50\% decrease of total tumour volume of measurable tumours or an increase in size/number of lesions, respectively. Outcome was considered to be a partial response (PR) when there was more than $50 \%$ decrease, but not complete disappearance of tumour, no new lesions occurred and at least partial improvement of clinical signs. Disappearance of all measurable tumours and clinical signs was considered as a complete response (CR). Response rates are defined as the percentage of cats with $\mathrm{CR}$ and PR. In order to be registered as responses, the responses should sustain at least for 3 weeks.

Toxicity was looked upon by scoring of AE. The AE scored were: vomiting, diarrhoea, fever, anorexia, obstipation, hair loss, dullness and pain (perception owner). Grading of these $\mathrm{AE}$ was done according to VCOG-CTCAEv1.0. ${ }^{11}$

Survival time of all cats was calculated as the interval from start of treatment till death of the subject or time at which the cat was last known to be alive. Only deaths due to ML or the treatment were counted as an event. DFP of cats which went into $\mathrm{CR}$ were calculated as the interval between moment of confirmation of CR and relapse or the date that the cat was last known to be free of disease, only counting relapses as events.

\section{Statistical analysis}

Survival curves (DFP and survival time) were drawn by the Kaplan-Meier method. Cats were censored in the survival analysis because they were either (1) lost to follow-up, (2) their death was not caused by ML or the treatment or (3) they were alive at the end of the study period. Censoring for DFP was done in case of (1) lost to follow-up, (2) death before occurrence of relapse or (3) relapse did not occur before the end of the study period. The variables age, sex, anatomic location, breed and prednisolone pretreatment were evaluated for predicting recurrence after $\mathrm{CR}$ and death using the log-rank test and univariate and multivariate Cox regression models. Differences among groups were evaluated by the nonparametric tests Mann-Whitney test and Kruskal-Wallis test when Gaussian distribution could not be assumed. Differences were considered to be significant at $P<0.05$. Statistical analysis of data was performed with SPSS $16.0^{\circledR}$ computer software.

\section{Results}

\section{Animal characteristics}

A total of 26 cats conformed all selection criteria and were included in this study. Of the 26 animals in this study, 16 were seen in the last 2 years. Half of the animals were male and 19 were of 
Table 2. Characteristics of the 26 cats included in the study

\begin{tabular}{llrr}
\hline Variable & \multicolumn{1}{c}{ Category } & $\boldsymbol{n}$ & \% \\
\hline Gender & Female intact & 2 & 7.7 \\
& Female spayed & 11 & 42.3 \\
& Male intact & 0 & 0.0 \\
Breed & Male castrated & 13 & 50.0 \\
& Domestic Shorthair & 19 & 73.1 \\
& Bengal & 1 & 3.8 \\
& Sphynx & 1 & 3.8 \\
& Norwegian Forrest Cat & 1 & 7.7 \\
Age & Russian blue & 2 & 3.8 \\
& British shorthair & 1 & 3.8 \\
& Siamese & 1 & 3.8 \\
Anatomical location & 4 & 15.4 \\
& <5years & 7 & 26.9 \\
& 5-10years & 15 & 57.7 \\
& Alimentary & 3 & 11.5 \\
& Liver & 2 & 7.7 \\
& Peripheral & 3 & 11.5 \\
& Nasal & 10 & 38.5 \\
& Mediastinal & 1 & 3.8 \\
& Miscellaneous & 7 & 26.9 \\
\hline
\end{tabular}

the Domestic Shorthaired breed (Table 2). No specific breed was significantly over-represented. Mean age was 9.5 years (median 10.4 years and range 2.1-14.3 years). No clear bimodal age distribution but rather a normal distribution was found. None of the 15 cats that were tested on FeLV and FIV were found to be positive for either one of the retroviruses. Ten cats had a medical history of corticosteroid therapy within 3 weeks prior to chemotherapy induction, 15 did not and for 1 cat there was no information available. In the classification system used in this study the nasal form was most common. Only one of the cat was diagnosed with the mediastinal form, a 2-year-old Siamese. Of the seven cats that were categorized in the group 'miscellaneous', three cats were diagnosed with a subcutaneous lymphoma, two with a laryngeal lymphoma, one with a tracheal and one with a retrobulbar lymphoma.

Mean age of the group with nasal lymphoma was 10.6 years (SD 2.5 years), mean age of the cats with peripheral lymphoma was 6.6 years (SD 2.6 years) and mean age of the cats with alimentary lymphoma was highest with 12.6 years (SD 1.8 years). All alimentary lymphomas were of the large cell type.

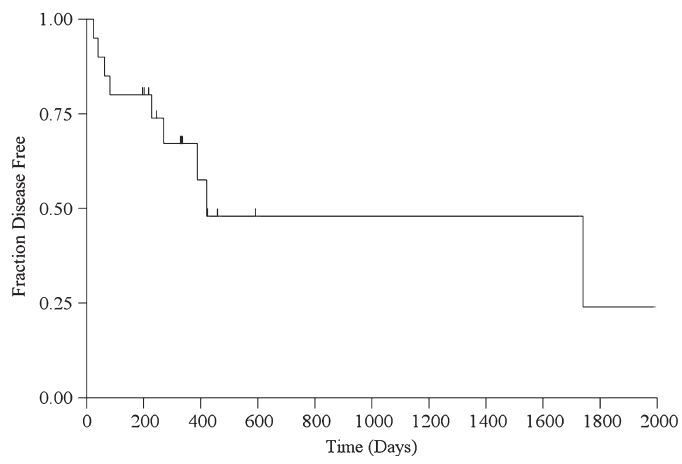

Figure 1. Disease free period (DFP) curve of the 20 cats that went into complete remission. Vertical bars represent censored observations. Estimated 1-year and 2-year DFP were 67.1 and $48.0 \%$, respectively. Median DFP was 421 days.

\section{Animal cooperation}

All cats could be restrained by neck lifting - in most with support of lower legs - during all administrations and no injury occurred to animal care takers or oncologists at any time. No canula dislodgment during therapy was encountered. Cats tolerated the procedure without any visible pain.

\section{Response to treatment}

Twenty animals (76.9\%) of the total 26 cats went into CR, 5 cats $(19.2 \%)$ reached a PR and 1 cat (3.8\%) had no significant response. Median DFP was 421 days, with a 1 -year estimated DFP of $67.1 \%$ and a 2-year estimated DFP of $48.0 \%$ (Fig. 1). Animals with alimentary lymphoma had the lowest DFP (228 days). The median DFP was highest for cats with nasal and peripheral lymphoma, 388 and 421 days, respectively. The 1-year DFP rate for cats with nasal lymphoma was $62.5 \%$. No significant differences in CR rates and DFP between neither anatomic types nor breeds were found. Exact time to reach remission could not be determined because cats were only seen weekly, but was less than 14 days for each of the individuals that reached CR. Total median survival was 388 days with a 1-year survival rate of $54.7 \%$ and a 2 -year survival rate of $46.9 \%$ (Fig. 2).

Of the 13 cats that had a relapse before the end of this study, 6 animals underwent a second treatment; 3 cats were treated with L-asparginase only, 2 with L-asparginase in combination with cytosar $^{\circledR}$ and 


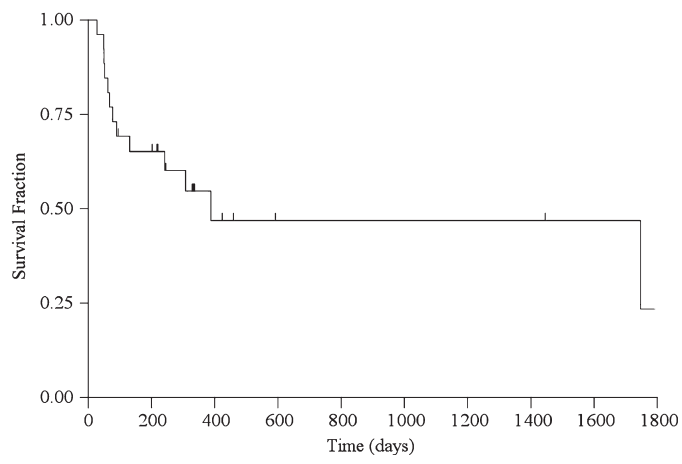

Figure 2. Survival curve for 26 cats with malignant lymphoma treated with IP-COP. Vertical bars represent censored observations. Estimated overall 1-year and 2-year survival rate was 54.7 and $46.9 \%$, respectively. Median survival time was 388 days.

1 was treated with L-asparginase and leukeran. None of these cats reached a second remission. One cat that underwent relapse (421 days after onset of treatment) underwent a second treatment with the IP-COP protocol and did reach a new CR with a new DFP of 1016 days while being treated continuously.

\section{Adverse events}

In general, this chemotherapy protocol was well tolerated by the cats. No therapy-related deaths occurred. Seven AEs were scored according to VCOG-CTCAEv1.0 terminology. ${ }^{11}$ Results are listed in Table 3. The grading system consists of five grades in which Grade 1 is mildest and Grade 5 is death. All AE except anorexia and hair loss were graded as either Grade 1 or Grade 2, being mild to moderate. No specific IP-administration related AE occurred.
Diarrhoea was seen only as Grade 1 or 2, Grade 1 being defined as an 'increase of $>2$ stools a day over baseline' and Grade 2 as an 'increase of 2-6 stools a day' or 'need of parenteral fluids'.

Vomiting when present was usually seen as Grade 1, less than three episodes of vomiting within 24 $h$. The cat that had one period of vomiting graded as Grade 2 had less than three episodes a day for 2-5 days, after the first treatment. The cat had been diagnosed with a gastrointestinal lymphoma.

Anorexia was usually seen as Grade 1 when there is only a need of dietary change to maintain appetite. In two cats, a Grade 2 anorexia was noted because of a decreased oral intake for 2-3 days without weight loss.

In one third of the cats one or more periods of a Grade 1 obstipation was noted, occurring only occasionally or intermitted and needing only sparse use of laxatives. In three cats, the obstipation became persistent and laxatives or enemas had to be used constantly and therefore the AE was graded as Grade 2.

Loss of hair or, more mildly, thinning of the coat was either localized, Grade 1 (e.g. only the vibrissae) or generalized (Grade 2 for thinning of the coat and Grade 3 for denuding).

Pain was only reported once by an owner, to whom the cat appeared to have a mild abdominal pain for only 1 day. It is graded as 1 which is defined as 'mild pain, not interfering with function'. By following IP treatments no pain was recorded anymore.

Several owners reported that cats were inactive and made a fatigue impression after therapy. This state was maintained for anywhere between 1 and 5 days. Grade 1 is defined as 'mild lethargy

Table 3. Adverse events in 26 cats with malignant lymphoma after receiving intraperitoneal chemotherapy

\begin{tabular}{|c|c|c|c|c|c|c|c|}
\hline \multirow[b]{2}{*}{ Adverse event } & \multirow[b]{2}{*}{ Total number } & \multicolumn{5}{|c|}{ Grade $^{a}$} & \multirow[b]{2}{*}{ Number of cats involved } \\
\hline & & 1 & 2 & 3 & 4 & 5 & \\
\hline Diarrhoea & 7 & 2 & 5 & 0 & 0 & 0 & $3(11.5 \%)$ \\
\hline Vomiting & 23 & 22 & 1 & 0 & 0 & 0 & $11(42.3 \%)$ \\
\hline Anorexia & 20 & 17 & 3 & 0 & 0 & 0 & $9(34.6 \%)$ \\
\hline Obstipation & 26 & 20 & 6 & 0 & 0 & 0 & $9(34.6 \%)$ \\
\hline Hair loss & 8 & 4 & 3 & 1 & 0 & 0 & $7(26.9 \%)$ \\
\hline Pain & 1 & 1 & 0 & 0 & 0 & 0 & $1(3.8 \%)$ \\
\hline Dullness/lethargy & 20 & 20 & 0 & 0 & 0 & 0 & $10(38.5 \%)$ \\
\hline
\end{tabular}

${ }^{\mathrm{a}}$ Grading was performed according to VCOG-CTCAEv 1.0 guidelines. 
over baseline' and Grade 2 as 'moderate lethargy causing some difficulty with performing ADL' $(\mathrm{ADL}=$ activities of daily living). Owner descriptions made us decide to grade this inactive state as Grade 1.

\section{Prognostic factors for the duration of remission and survival}

Five pretreatment parameters were evaluated for their prognostic predictive value for the duration of survival and remission. Of these parameters only age appeared to be prognostic for DFP length and only when categorized in age-groups of 5 years (i.e. $0-5$ years, $5-10$ years and $10-15$ years). It appeared that the youngest group had the longest DFP $(P=0.046)$. Including other parameters in a multivariate analysis did not lead to conversion, so they were excluded from the analysis. Survival times also were significantly higher for the youngest group of individuals $(P=0.031)$. No other prognostic parameter was significant in predicting either DFPlength or survival. Response to therapy (reaching CR) was significant in predicting length of survival but cannot be seen as a prognostic parameter $(P=0.002)$.

\section{Discussion}

This study was commenced to prove safety and efficacy of the IP-COP protocol used at our department because of practical, safety and ethical reasons. Administration is faster, venapuncture is not needed and therefore there is no risk for perivascular injection. Because of the shortness of the procedure stress for the cat is minimal and overly aggressive or frightened cats do not need to be sedated or anaesthetized. Furthermore, the risk for the administrator of getting bitten or scratched is minimal even in aggressive animals when the animal is fixed properly by the veterinary assistant.

In veterinary literature, we have not been able to find any report describing the IP use of chemotherapeutic agents in cats. In human medicine, however, IP use of these agents is no exception. It is used for treatment of patients with certain types of tumour in the peritoneal cavity (e.g. ovarian cancer or colon cancer). ${ }^{12}$ The fundamental goal of this route of administration in humans is creating a locally high dose of the chemotherapeutic drug at the site of the tumour for a longer period of time while reducing systemic toxicity. Indeed, the IP administration of several chemotherapeutic drugs has proven to be safer whilst still effective. Survival rates of combined IV and IP therapy were higher than IV therapy alone. ${ }^{12,13}$

When treating feline ML outside the peritoneal cavity a locally high dose in this cavity is not the primary goal. Since effects of chemotherapeutics are dose- but mostly time-dependent, prolonged and sufficient high systemic levels of chemotherapeutics (and/or active metabolites) is what one should aspire. In 2005, Miyagi et al. studied the 24h AUC (area under curve) for carboplatin after IP and IV administration. These 24-h AUC were exactly the same for both routes so they made the very promising conclusion that the IP route can be used for systemic therapy. ${ }^{14}$ Extrapolation of results obtained in human studies is hard, mainly because other drugs are used and often in much higher concentrations. Lack of knowledge of the pharmacokinetic values of chemotherapeutics used in cats make predicting parameters like bioavailability very difficult. For this further pharmacodynamic studies have to be conducted.

In our study, the nasal form of ML was most often diagnosed. In a previous study at our institute, the mediastinal form was most common and nasal lymphoma only accounted for $13 \% .{ }^{1}$ So a certain shift in anatomical location has taken place. This increase in nasal lymphoma has been reported elsewhere. ${ }^{3}$ There seems to be an overall increase in patients with ML presented at our faculty. Most of the cats in this study began treatment in 2009 and 2010. This increase in incidence was also reported by elsewhere. ${ }^{2}$ Possibly more owners are willing to treat their cats for ML.

The mean age of 9.5 years reported in our study is consistent with ages reported elsewhere, ${ }^{15-17}$ but higher than found in 2002 and earlier studies. ${ }^{8,18}$ The big difference in both age and anatomical form can be explained by the large group of young Siamese cats present in the earlier study. Siamesetype cats are known to be genetically predisposed for the development of mediastinal lymphoma. ${ }^{1,2}$ Several studies report a bimodal age distribution 
for cats with ML. ${ }^{1,2,16}$ The first peak (i.e. 1-2 years) used to be explained by a FeLV infection leading to $\mathrm{ML}$ in young animals, often in Siamese-type breeds. This seems to be incorrect. ${ }^{1,17}$ Even when there are hardly any FeLV positive cats a bimodal distribution is seen. ${ }^{1,3}$ Other aetiologic factors must play a role. In the study presented here, no clear bimodal age distribution was apparent, rather a normal distribution. Absence of a large group of young Siamese - with only one in the current study - may account for this. It is obvious that FeLV plays a subordinate role in the population treated in this study. This is in concurrence with the earlier studies. ${ }^{1,4,15}$ In a recent study none of the antigen-negative cats with lymphoma was detectably infected with latent provirus FeLV, thereby making a possible role of FeLV in our study even further unlikely. ${ }^{19}$

The original COP protocol has already proven to be effective. ${ }^{1,5,8,20}$ In this study, the median overall survival time (388 days) was higher than reported in an earlier study from our institute $(266 \text { days })^{1}$ and also higher than reported with other chemotherapy protocols, like the University of Wisconsin-Madison and VCM protocols (both 210 days), ${ }^{16,18}$ and much higher than a single doxorubicin protocol (84 days). ${ }^{21}$ The 1-year and 2year survival rates were 54.7 an $46.9 \%$, respectively, comparable to the 48.7 and $39.9 \%$, respectively, reached in our earlier study. ${ }^{1}$

Assessment of the remission rate in this study shows a comparable result as previous COP protocol studies. ${ }^{1,8}$ Reports of remission rates vary between 29 and $79 \% .^{3}$ In this study, the CR rate was $76.9 \%$, comparable to the $75.4 \%$ in our earlier study. ${ }^{1}$ This is higher than achieved by other protocols, like Wisconsin-Madison protocols with $47 \%$, VCM (vincristine, cyclophosphamide and methotrexate) with $62 \%$ and single agent doxorubicin with $26 \%$, respectively. ${ }^{16,18,21}$ But also higher than the one Moore et al. achieved using a COP protocol, ${ }^{20}$ illustrating that outcome of treatment is dependent on much more than solely the protocol used; anatomical site, age of the cat, immunological type and many more factors may play a role.

Duration of complete remission (i.e. DFP) exceeds that of most other studies. Median DFP was 421 days, compared to 251 days of the IV-COP protocol of Teske et al. in 2002, and 264 and 281 days of IV-COP protocols using other chemotherapeutics in their maintanance. ${ }^{1,7,20}$ Single agent protocols often have substantially lower DFP (e.g. 92 days). ${ }^{21}$ Median DFP was only exceeded by Milner et al. who used a University of WisconsinMadison protocol (654 days). In this study, however, 7 of the 38 cats had a small cell intestinal lymphoma, a form which has a much better prognosis. ${ }^{16}$ Estimated 1-year DFP was $67.1 \%$ and estimated 2-year DFP was $48.0 \%$, depicting a fairly stable remission after discontinuation of therapy at 1 year. Yet again these figures are somewhat higher than previous results (51.4 and $37.8 \%$, respectively), ${ }^{1}$ other studies did not report these rates. Taken all treatment results together, this study demonstrates the IP-COP protocol to be at least as effective as the IV-COP protocol and the other reported protocols.

Peripheral lymphoma (421 days) and nasal lymphoma (388 days) had longest median DFPs. In previous studies cats with alimentary lymphoma had poorest remission and survival time. ${ }^{1,7,8}$ Although not significant, this study seems to display the same tendency. The mediastinal form most often responds well to the COP protocol but less so using other protocols. ${ }^{1,6,8}$ In conclusion; prognosis for nasal and peripheral lymphoma is good and prognosis for the alimentary form is poorer using the COP protocol, either IV or IP.

The finding that young-aged cats are more likely to have a longer DFP and longer survival periods is noteworthy. This was the only significant prognostic factor for a favourable outcome found in this study even when other factors were included in a multivariate model. Age as prognostic factor has also been identified by Malik et al. ${ }^{4}$ Possible explanations lie in difference in tumour type between younger and older animals, although no significant difference in mean age of the different types of MLs were found in this study and also immunophenotype has not been proven prognostic in earlier studies. $^{22,23}$ Other aetiologic factors may play a role in young animals to make chemotherapy more effective. Pretreatment with corticosteroids did not negatively influence outcome in the cats in this study. This is in contrast to dogs where it is negatively associated with treatment results. ${ }^{24} \mathrm{~A}$ certain 
predictive value cannot be excluded, however, since there is wide variation in corticosteroid treatment in the cats that underwent prior corticosteroid therapy by private practitioners. Breed, ${ }^{1}$ anatomic site, ${ }^{7,8}$ FeLV status ${ }^{6,16}$ and stage of disease ${ }^{6,18}$ are variables shown to have a prognostic value in other studies. The fact that we could not identify these variables to be prognostic in our study can be due to the limited numbers of animals. Although not a real prognostic factor in the narrow sense, the most important indicator for reaching long remission and survival times was achieving a CR $(P=0.01)$. Not reaching CR should be regarded as treatment failure. ${ }^{1,6,8,16}$

We experienced that once relapse occurred during first treatment a second treatment had poor efficacy. Second treatment was conducted with other chemotherapeutics (i.e. L-asparginase, cytarabine, chlorambucil) than used in the COPprotocol. Hence, tumours in these individuals reached certain state of resistance in which none of the chemotherapeutics we used were effective anymore. Remarkably one cat that underwent relapse 2 months after the 1 -year treatment protocol reached a new $\mathrm{CR}$ in 2 days through resumption of the IP-COP protocol and stayed in remission for almost 3 years.

Foremost we want to report that no special AE related to IP administration were reported nor seen by the clinicians. The majority of all reported $\mathrm{AE}$ was graded as mild (i.e. Grade 1). Obstipation had the largest total number of episodes, but was only reported in nine animals. Only a few of them needed treatment with laxatives. Not a single cat showed signs of haemorrhagic cystitis. Vomiting was seen in 11 animals with a total of 23 episodes where 22 were graded as Grade 1. A mild anorexic state could almost always be counteracted by a change of menu. Owners of ten cats reported a certain dullness or lack of energy during a 2-3 day period following drug administration. Loss of hair was most often limited to loss of vibrissae. These numbers might seem high from a first point of view, but are relatively low compared to the number of drug administrations performed during the course of this study. Furthermore, none of the owners wished to stop therapy because of AE. So severity can be considered low enough on behalf of the animals (under given circumstances) and incidence is low enough for owners to tolerate. It is hard to compare these results with other studies because none of them used this grading system. Other studies reported a fair degree of gastrointestinal toxicosis. ${ }^{16,20,21}$ Once again, from our point of view one of the most important findings is the lack of any specific IP-administration related AE. Besides the clinical efficacy it is found that owners are generally very satisfied with their choice of commencing chemotherapy with the COP protocol. ${ }^{25,26}$

Limitations of this study include the small study group, very low case numbers in certain groups (e.g. mediastinal lymphoma or exotic breeds), lack of a control group and the possible biases in case selection. The low numbers result in lack of power to reach significance and make it hard to execute a reliable multivariate analysis. Another difficulty in a retrospective study as presented is the difficulty of interpreting and verification of the accuracy of the case reports. Grading AE was hard because we had to rely on owner reports. Furthermore, it should be stated one more time that extrapolation of obtained results to other populations is very hard, mainly because of big differences in genetics and retrovirus incidence.

Although this study might give the impression that this technique of administration of vincristine and cyclophosphamide is exquisitely easy and 'ready-to-use' for anyone no matter what experience they may have in chemotherapy handling, a serious warning has to be given. The IP administration was performed by experienced oncologists in a situation where every safety aspect of good veterinary practice (GVP) of preparation and administration of cytostatic drugs was executed in line of the ECVIM-CA guidelines in order to avoid contamination of drugs. ${ }^{27}$

The main goal of this study was to investigate the feasibility of IP administration, the efficacy and lack of AE. Results seem to indicate that the IP-COP protocol is indeed an effective one. Because of the mild AE, AE quantitatively comparable to the IV protocols and an absence of real IP-related AE we feel safe to say that it can be seen as an equally safe alternative for the IV administration. The next step we will take in proving efficacy and safety of this new route of administration is a controlled pharmacokinetic experiment in which we assess 
serum levels of chemotherapeutics both after IV and IP administration.

\section{References}

1. Teske E, van Straten G, van Noort R and Rutteman GR. Chemotherapy with cyclophosphamide, vincristine, and prednisolone (COP) in cats with malignant lymphoma: New results with an old protocol. Journal of Veterinary Internal Medicine 2002; 16: 179-186.

2. Louwerens M, London CA, Pedersen NC and Lyons LA. Feline lymphoma in the post-feline leukemia virus era. Journal of Veterinary Internal Medicine 2005; 19: 329-335.

3. Hayes A. Feline lymphoma 1. Principles of diagnosis and management. In Practice 2006; 28: 516-524.

4. Malik R, Gabor LJ and Canfield PJ. Lymphoma in Australian cats-lessons for Europe? Journal of Feline Medicine and Surgery 2003; 5: 147-150.

5. Ettinger SN. Principles of treatment for feline lymphoma. Clinical Techniques in Small Animal Practice 2003; 18: 98-102.

6. Vail DM, Moore AS, Ogilvie GK and Volk LM. Feline lymphoma (145 cases): proliferation indices, cluster of differentiation 3 immunoreactivity, and their association with prognosis in 90 cats. Journal of Veterinary Internal Medicine 1998; 12: 349-354.

7. Simon D, Eberle N, Laacke-Singer L and Nolte I. Combination chemotherapy in feline lymphoma: treatment outcome, tolerability, and duration in 23 cats. Journal of Veterinary Internal Medicine 2008; 22: 394-400.

8. Cotter SM. Treatment of lymphoma and leukemia with cyclophosphamide, vincristine and prednisolone: II. Treatment of cats. Journal of the American Animal Hospital Association 1983; 19: $166-172$.

9. Los G, Mutsaers PHA, van der Vijgh WJF, Baldew GS, de Graaf PW and MeVie JG. Direct diffusion of cfV diamminedichloroplatinum (II) in intraperitoneal rat tumors after intraperitonal chemotherapy: a comparison with systemic chemotherapy. Cancer Research 1989; 49: 3380-3384.

10. Markman M. Intraperitoneal chemotherapy. Critical Reviews in Oncology: Hematology 1999; 31: 239-246.

11. VCOG. Veterinary Co-Operative Oncology Group-common terminology criteria for adverse events (VCOG-CTCAE) following chemotherapy or biological antineoplastic therapy in dogs and cats. Veterinary Comparative Oncology 2004; 2: 194-213.
12. Markman M. Intraperitoneal antineoplastic drug delivery: rationale and results. The Lancelet Oncology 2003; 4: 277-283.

13. Markman M. Intraperitoneal chemotherapy. In: Perry MC (ed). The chemotherapy source book. $4^{\text {th }}$ ed. Philadelphia, PA, Lippincott Wilkins \& Williams. 2007:75-77.

14. Miyagi $Y$, Fujiwara K, Kigawa J, Itamochi H, Nagao S, Aotani E, Terakawa N, Kohno I and Sankai Gynecology Study Group (SGSG). Intraperitoneal carboplatin infusion may be a pharmacologically more reasonable route than intravenous administration as a systemic chemotherapy. A comparative pharmacokinetic analysis of platinum using a new mathematical model after intraperitoneal vs. intravenous infusion of carboplatin - a Sankai Gynecology Study Group (SGSG) study. Gynecologic Oncology 2005; 99: 591-596.

15. Gabor LJ, Jackson ML, Trask B, Malik R and Canfield PJ. Feline leukemia virus status of Australian cats with lymphosarcoma. Australian Veterinary Journal 2001; 79: 476-481.

16. Milner RJ, Peyton J, Cooke K, Fox LE, Gallagher A, Gordon $\mathrm{P}$ and Hester J. Response rates and survival times for cats with lymphoma treated with the University of Wisconsin-Madison chemotherapy protocol: 38 cases (1996-2003). Journal of the American Veterinary Medical Association 2005; 7: 1118-1122.

17. Court EA, Watson AD and Peaston AE. Retrospective study of 60 cases of feline lymphosarcoma. Australian Veterinary Journal 1997; 6: 424-427.

18. Mooney SC, Hayes AA, MacEwen EG, Matus RE, Geary A and Shurgot BA. Treatment and prognostic factors in lymphoma in cats: 103 cases (1977-1981). Journal of the American Veterinary Medical Association 1989; 194: 349-354.

19. Stützer B, Simon K, Lutz H, Majzoub M, Hermanns W, Hirschberger J, Sauter-Louis C and Hartmann K. Incidence of persistent viraemia and latent feline leukaemia virus infection in cats with lymphoma. Journal of Feline Medicine and Surgery 2011; 13: 81-87.

20. Moore AS, Cotter SM, Frimberger AE, Wood CA, Rand WM and L'Heureux DA. A comparison of doxorubicin and COP for maintenance of remission in cats with lymphoma. Journal of Veterinary Internal Medicine 1996; 10: 372-375.

21. Kristal O, Lana SE, Ogilvie GK, Rand WM, Cotter SM and Moore AS. Single agent chemotherapy with doxorubicin for feline lymphoma: a retrospective 
study of 19 cases (1994-1997). Journal of Veterinary Internal Medicine 2001; 15: 125-130.

22. Patterson-Kane JC, Perrins Kugler B and Francis K. The possible prognostic significance of immunophenotype in feline alimentary lymphoma: a pilot study. Journal of Comparative Pathology 2004; 130: 220-222.

23. Gabor LJ, Canfield PJ and Malik R. Immunophenotypic and histological characterization of 109 cases of feline lymphosarcoma. Australian Veterinary Journal 1999; 77: 436-441.

24. Piek CJ, Rutteman GR and Teske E. Evaluation of the results of a L-asparaginase-based continuous chemotherapy protocol versus a short doxorubicin-based induction chemotherapy protocol in dogs with malignant lymphoma. Veterinary Quarterly 1999; 21: 44-49.
25. Tzannes S, Hammond MF, Murphy S, Sparkes A and Blackwood L. Owners 'perception of their cats' quality of life during COP chemotherapy for lymphoma. Journal of Feline Medicine and Surgery 2008; 10: 73-81.

26. Brønden LB, Rutteman GR, Flagstad A and Teske E. Study of dog and cat owners' perceptions of medical treatment for cancer. Veterinary Record 2003; 152: $77-80$.

27. ECVIM-CA. Preventing Occupational and Environmental Exposure to Cytotoxic Drugs in Veterinary Medicine, $2^{\text {nd }}$ edn., 2007. http://www. ecvim-ca.org/index.php?option=com_content\& view $=$ article\&id $=72 \&$ Itemid $=70$ [accessed 11 March 2012]. 